\title{
Orient extrême : deux adaptations musicales de King Lear et Hamlet au Japon
}

\section{Claire Bardelmann}

\section{(2) OpenEdition}

\section{Journals}

\section{Édition électronique}

URL : http://journals.openedition.org/shakespeare/1515

DOI : 10.4000/shakespeare.1515

ISSN : 2271-6424

\section{Éditeur}

Société Française Shakespeare

\section{Édition imprimée}

Date de publication : 13 décembre 2009

Pagination : 159-174

ISBN : 2-9521475-6-6

\section{Référence électronique}

Claire Bardelmann, «Orient extrême : deux adaptations musicales de King Lear et Hamlet au Japon », Actes des congrès de la Société française Shakespeare [En ligne], 27 | 2009, mis en ligne le 13 décembre 2009, consulté le 06 mai 2019. URL : http://journals.openedition.org/shakespeare/1515 ; DOI :

10.4000/shakespeare.1515 


\title{
Shakespeare et l'Orient
}

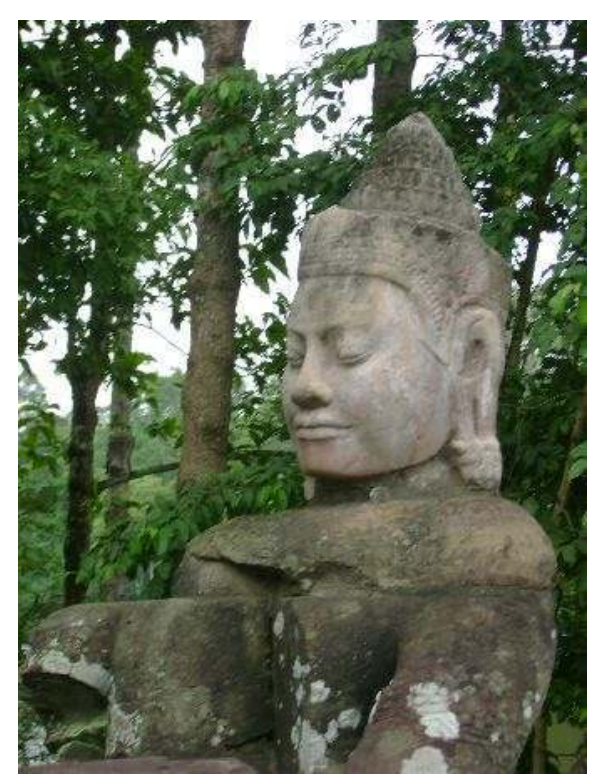

\author{
actes du Congrès \\ organisé par la \\ les 12, 13 et 14 mars 2009 \\ textes réunis par \\ Pierre KAPITANIAK \\ sous la direction de \\ Jean-Michel DÉPRATS
}

SOCIÉTÉ FRANÇAISE SHAKESPEARE 
COUVERTURE :

photo J.-M. Déprats 2008

conception graphique et logo

Pierre Kapitaniak

\section{(C) 2009 Société Française Shakespeare \\ Institut du Monde Anglophone \\ Université de Paris III - Sorbonne Nouvelle \\ 5 rue de l'École de Médecine 75006 Paris \\ www.societefrancaiseshakespeare.org}




\title{
ORIENT EXTRÊME : DEUX ADAPTATIONS MUSICALES DE KING LEAR ET HAMLET AU J APON
}

\author{
Claire BARDELMANN
}

Le renouveau de l'intérêt pour Shakespeare au Japon dans les années soixante est le prélude à plusieurs adaptations musicales de ses œuvres, notamment les versions en rock opéra de Hamlet et de King Lear. Les trois adaptations très libres de Hamlet par des groupes de rock et de heavy metal, dans les années 1980, ainsi que l'opéra expérimental RiaO tiré de King Lear par le célèbre poète Terayama Shûii (1935-1983), ne témoignent pas seulement d'un phénomène de translation culturelle extrême, qui participe d'ailleurs à la définition du "nouveau théâtre " japonais. Le choix de Hamlet, vu comme tragédie d'une errance existentielle, et de King Lear, interprété comme forme de nihilisme moderne, montrent que les œuvres de Shakespeare servent aussi une réflexion sur les mutations infligées à la société et aux valeurs traditionnelles japonaises par la montée du capitalisme dans l'après-guerre et surtout à partir des années 1960. Cette opposition se traduit par le traitement musical et la chorégraphie adoptés par des auteurs et compositeurs alternatifs connus pour leur utilisation de la provocation - violence et érotisme. Mais cette volonté d'accompagner le bouleversement des modèles se mesure aussi à l'insertion de références à des composantes traditionnelles de la culture japonaise comme le culte Aum Shinri-kyo, le théâtre nô, ou l'utilisation rituelle de danseurs buto.

Renewed interest in Shakespeare in Japan in the 1960s led to several musical adaptations of his works, including rock opera versions of Hamlet and King Lear. The three adaptations of Hamlet in rock and heavy metal music in the 1980s and Terayama Shûji's (1935-1983) experimental opera RiaO (after King Lear) belong to the Japanese "new drama" and are examples of extreme cultural translations. But they also pinpoint how Shakespeare's works implement a reflection on socio-cultural transformations in post-war Japan, especially from the 1960s, when traditional values were challenged by the rise of capitalism. RiaO and Rock Opera Hamlet reflect the opposition to such mutations through the handling of choreography and music by underground authors known for their use of violence and eroticism. The challenging of traditional models is also expressed through the many references to traditional components of Japanese culture, such as Noh drama or Buto dancers.

$\mathrm{I}$

l existe au Japon une ancienne et importante tradition shakespearienne. L'œuvre dramatique de Shakespeare est jouée depuis 1886, la plupart du temps dans des adaptations. Le seul Hamlet a donné lieu depuis cette date à plus de 135 mises en scènes différentes ${ }^{1}$. De plus, le théâtre de Shakespeare a connu un rôle de premier plan comme creuset de l'expérimentation théâtrale japonaise

${ }^{1}$ Décompte effectué d'après la liste établie dans J. R. Mulryne, Takashi Sasayama (éds) Shakespeare and the Japanese Stage, Cambridge, C.U.P., 1998, p. 257-331. Voir aussi Takeshi Murakami, "Shakespeare and Hamlet in Japan : A Chronological Overview ", in Uéno Yashiko (éd), Hamlet and Japan, New York, AMS Press, 1995, 239-241. Cette tragédie a été la première pièce de Shakespeare adaptée au Japon (en 1886, en style kabuki classique, par Robun Kanagaki) ; et de 1886 à 1994, on ne dénombre pas moins de 135 mises en scène, sans préjudice des nombreuses œuvres littéraires en tout genre inspirées par la tragédie (romans à la première personne, pièces de théâtre, romans, romans policiers, nouvelles, poésie...). King Lear ne fait pas partie des pièces de Shakespeare les plus diffusées au Japon (après Hamlet, ce sont plutôt The Merchant of Venice, Romeo and Juliet), mais sur la même période elle a tout de même été représentée 57 fois. 
et de l'évolution des formes, depuis les premières adaptations de Shakespeare en kabuki jusqu'aux mises en scène occidentalisées du théâtre shingeki. Ainsi, le kabuki, qui est un genre de théâtre traditionnel, a été le principal agent d'expérimentation en matière de théâtre shakespearien avant l'avènement du théâtre shingeki (théâtre à l'occidentale fondé au début du $\mathrm{xx}^{\mathrm{e}}$ siècle) : en effet, le kabuki a connu une constante évolution de son répertoire et de ses techniques au fil des siècles, ce qui explique la plasticité avec laquelle ce théâtre a accueilli la pratique théâtrale occidentale, et la souplesse avec laquelle il a su faire représenter les tensions du monde nippon par le frottement des cultures. James C. Brandon remarque à ce propos :

Throughout Kabuki's history, new types of plays and performing techniques had been devised to express the concerns of each new era. [...] Therefore, when playwrights and actors of the Meiji period created living history and cropped-hair plays, they were continuing the usual process in Kabuki of molding play forms to suit contemporary audiences. Kabuki actors and managers examined western theatrical practices to see what they might adopt. [...] Playwrights turned to European drama for ideas, situations, and characters, and even to scenes and whole plays that they thought could be adapted to the Kabuki stage ${ }^{2}$.

Les adaptations musicales de Shakespeare au Japon illustrent particulièrement le regain d'intérêt pour Shakespeare depuis la fin des années 1960. Comme l'explique Kazuko Hasebe dans un article de 1974 :

Recent Shakespeare translations and stage productions in Japan are increasing in number and variety. From the early Meiji period of free adaptation up to the present Shakespeare has grown in popularity, enchanting the Japanese reading public and audience ${ }^{3}$.

Les comédies musicales adaptées de Shakespeare depuis 1970 illustrent bien cette renaissance shakespearienne. Il y en a eu huit, des "Amants de Vérone » (Berôna no koibitotachi) en 1971 à la version rock de Hamlet par le groupe X-Japan (1996). Parmi ces œuvres,

\footnotetext{
2 James C. Brandon, «Shakespeare in Kabuki », in Performing Shakespeare in Japan, éd. Minami Ryuta, Ian Carruthers, John Gillies, Cambridge, C.U.P., 2001, p. 36-37.

3 Kazuko Hasebe, « Recent Shakespeare Translations and Stage Productions in Japan », in Shakespeare Translation, Tokyo, Yushodo Shoton LTD, 1978, p. 61. Voir aussi Hiroshi Yamamoto, "The Current 'Shakespeare Boom' in Japan ». Renaissance Bulletin 4 (1977), p. 1-10.
} 
quatre sont des adaptations de $\mathrm{Hamlet}^{4}$, et une seule, RiaO, est tirée de King Lear 5 .

Parmi ces adaptations musicales, il en est deux qui sont représentatives de courants d'interprétation aux antipodes. La première, RiaO, relève du théâtre expérimental et s'inscrit dans le courant avant-gardiste du «nouveau théâtre » japonais. C'est une œuvre confidentielle destinée à un public de connaisseurs, et qui a d'ailleurs été donnée à Tokyo en 1991 lors du Festival International Shakespeare, d'où l'existence d'un synopsis en anglais destiné au public anglophone. L'autre œuvre, Rock Opera Hamlet, du groupe de hard rock japonais Penicillin, est une mise en scène grand public, écrite en japonais familier, et dont le langage scénique et musical est également populaire.

À première vue, ces deux œuvres ont pour seuls points communs d'être contemporaines, musicales, et de proposer une libre interprétation de l'original shakespearien. Pourtant, ces adaptations constituent toutes deux, à travers Shakespeare, des points de rencontre moderne entre le Japon et l'Occident. D'abord parce qu'elles effectuent un étonnant mélange des genres, des styles et des formes. Ensuite parce qu'à travers cette recherche, Shakespeare s'avère aussi un outil de questionnement du Japon moderne.

Le travail d'ajustement culturel dans RiaO et Rock Opera Hamlet se perçoit d'abord du point de vue du genre. Les deux œuvres se classent comme comédies musicales: Rock Opera Hamlet y appartient dans la mesure où l'opéra rock est un sous-genre de la comédie musicale. De son côté, RiaO est décrit comme " experimental musical » par Douglas Kerr, dans un article qui propose une analyse approfondie de $\mathrm{RiaO}^{6}$.

\footnotetext{
${ }^{4} 1980$ : adaptation en comédie musicale rock par Odashima Yushi (traduction), Nakajima Azusa et Sato Hirofumi (adaptation), Fuji Tomoroni (mise en scène), au Nagano Sun Plaza. 1982 : adaptation en théâtre nô de Hamlet en anglais par Munakata Kuniyoshi et le Noh Shakespeare Group. 1990 (reprise en 1991) : Hamlet no Jikkan, "The Time of Hamlet », opéra d'Odashima Yushi (traduction), Kato Tadashi (adaptation et mise en scène), Hayashi Hikaro et Hagi Kyoto (musique). 1993: Rock Opera Hamlet, de Matsumoto Kazuki (adaptation), Sato Hirofumi (mise en scène), Nakano Sun Plaza. Décompte effectué d'après Shakespeare and the Japanese Stage, p. 257-331.

${ }^{5}$ L'article de Kazuko Hasebe propose aussi une liste d'adaptations de Shakespeare au Japon depuis 1886 (art. cit., p. 61).

${ }^{6}$ Douglas Kerr, « King Lear as an Experimental Musical: The Japanese Production of RiaO », New Theatre Quarterly (2002), 18:2, p. 161-175.
} 
Mais dans les deux cas, ces définitions peinent à rendre compte de l'extraordinaire juxtaposition des formes, des genres dramatiques, des styles musicaux. Juxtaposition qui donne leur caractère surprenant à ces interprétations, comme en témoigne la première chanson de Rock Opera Hamlet, intitulée "Sword to Soul ». Hamlet a vu le fantôme de son père, et confie sa colère contre Claudius à Horatio. La chanson correspond schématiquement à I.v, où Hamlet s'exclame à propos de Claudius «O villain, villain, smiling, damned villain!», puis au dialogue entre Hamlet et Horatio à la fin de I.v, où Horatio remarque l'égarement de Hamlet: «these are but wild and whirling words, my lord».

Cette chanson se compose de deux parties au style très différent, qui traduisent toutes deux l'emportement : une courte introduction où le bruit d'un galop de cheval se prolonge par une citation de l'Étude en do mineur, opus $10, \mathrm{n}^{\circ} 12$, de Chopin. De la musique heavy metal succède à cette introduction; il s'agit du style dominant dans Rock Opera Hamlet. L'effet de contraste, délibérément très violent, provient de la confrontation brutale de deux types de musique européenne une pièce pour piano romantique, et de la musique populaire de variétés. C'est une constante de l'œuvre, qui présente une palette d'emprunts très bariolée, et qui fait appel à des époques, genres et styles de l'histoire musicale européenne entre lesquels existent d'énormes écarts. On trouve ainsi une introduction instrumentale en écriture atonale, des références à la musique sacrée, un passage en contrepoint ; et l'utilisation d'un choral, même si son traitement n'est pas canonique.

Les emprunts ne s'arrêtent pas à la musique, et c'est ce qui montre que le Hamlet de Shakespeare sert en fait de vecteur à une culture occidentale perçue de façon syncrétique, qui n'a évidemment plus aucun point commun avec l'Angleterre de Shakespeare. On trouve ainsi des références appuyées au catholicisme : une des chansons n'est autre que le «Salve Regina », chanté intégralement en latin sur fond de musique rock. Autres exemples : l'utilisation de l'orgue (même s'il est électronique) et de cloches d'église, et les citations du Dies Irae dans l'introduction de deux des chansons. Enfin, l'opéra rock fait des emprunts à des langues européennes : le latin, mais aussi l'anglais, pour les noms des personnages, et même le français : l'introduction d'une des chansons commence par les mots (parlés, et non chantés) 
« tu savais que c'était moi », susurrés en boucle par une voix féminine sur tapis de cordes synthétiques.

Ce kaléidoscope de citations, d'emprunts, d'influences, est là pour sa charge culturelle plus que pour son intérêt musical. Ils illustrent l'analyse que Carolyn S. Stevens fait des emprunts aux langues étrangères dans la musique pop japonaise: ce qu'elles communiquent est d'ordre esthétique et non d'ordre linguistique.

The integration of foreign languages into Japanese pop music demonstrates meaning on a number of levels; this fusion combines aesthetic ideas regarding both sound and image. [...] Whether a love or message song, serious or playful, foreign terms add value to the end product ${ }^{7}$.

Le mimétisme culturel de Rock Opera Hamlet, qui à première vue utilise majoritairement un idiome musical international (hard rock), est donc trompeur, puisque son esthétique s'appuie sur la notion d'écart culturel. Et l'on devrait d'autant plus parler de syncrétisme que Rock Opera Hamlet conserve des éléments typiquement japonais.

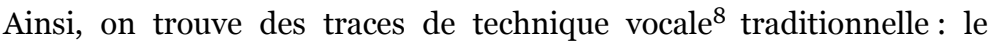
chanteur principal, Hakuei, qui joue Hamlet, mais aussi les autres chanteurs et les chœurs, utilisent dans les moments de grande tension le style jigoe, où le resserrement des cordes vocales produit une voix claire et nasalisée, surtout dans les vibratos 9 . À plusieurs reprises, ils recourent aussi à une technique d'écriture inspirée du nori, ornementation traditionnelle dans laquelle une déclamation est superposée à un passage de musique instrumentale entre deux phrases ou vers chantés. La chanson qui suit le solo « To Be or Not to be » $\left(\mathrm{n}^{\circ} 8\right.$

7 Carolyn S. Stevens, Japanese Popular Music: Culture, Authenticity and Power. Routledge, 2008, p. 123, p. 136.

${ }^{8}$ Comme la plupart des chanteurs de musique populaire contemporains. Voir Carolyn S. Stevens, op. cit., p. 23 : « for most Japanese male vocalists in the contemporary pop scene, vocal stylings are more inspired by Elvis Presley, Mick Jagger, and Bob Dylan than any classical models from premodern indigenous traditions. [...] But a discussion of traditional musical practice when analyzing contemporary pop is important [...] to move forward a sense of continuity in practice between traditional and contemporary musical forms, moving away from the binary opposition between old and new, Japanese and Westernized. »

${ }^{9}$ Le recours à cette technique n'est pas si courant, car, comme le souligne Teruma Shimizu, la technique et le style vocaux de la musique populaire japonaise issue du rock anglo-saxon suivent de près le modèle occidental. « A musicological analysis of melodies and singing in Japanese neo-rockabilly. » Popular music : Intercultural interpretations, 1998, 357-365. 
de l'opéra) permet ainsi d'entendre une belle citation du Dies Irae. Le morceau comporte une introduction instrumentale avec bruit de pluie et citation à l'orgue électronique du Dies Irae, puis une section centrale où se superposent musique instrumentale et déclamation, qui alternent avec le chant confié au soliste puis au chœur. La nasalisation est particulièrement perceptible dans les vibratos, notamment chez la soliste qui joue Ophélie et qui chante dans un registre suraigu.

Si Rock Opera Hamlet témoigne d'une stratification culturelle, alors que dire de celle de RiaO ? Sa complexité est la raison pour laquelle, du point de vue de la poétique, RiaO résiste à une catégorisation simple. Dans cette œuvre, le cadre est celui du théâtre : encore faut-il parvenir à le définir.

Théâtre musical, certes. Mais le terme utilisé par Douglas Kerr de « comédie musicale » semble inapproprié. La poétique européenne, en effet, opère une distinction entre les genres du théâtre et ceux du théâtre en musique (dont la comédie musicale), mais la poétique japonaise est tout autre, dans la mesure où le théâtre japonais traditionnel (nô, kabuki, bunraku) est un théâtre musical. Or, c'est précisément le cadre utilisé par RiaO. On peut donc préférer le terme de «théâtre expérimental », qui recouvre mieux le caractère kaléidoscopique de l'œuvre.

Une séquence du début de $\mathrm{RiaO}^{10}$, intitulée « Father Time Truth is the Daugther of Time », permet de mettre en évidence cette expérimentation avec une particulière netteté pour un public occidental. Elle correspond à I.i, la scène où Lear partage le royaume entre ses filles. Il s'agit de la quatrième séquence de RiaO. C'est aussi un des passages où la tragédie de Shakespeare est bien reconnaissable en termes d'intrigue et de texte. Pour un public européen familier de son œuvre, cette séquence souligne donc bien par contraste les éléments japonais traditionnels.

Le passage commence par une tirade de Regan, qui correspond à la tirade " I am alone felicitate / In your dear highness' love », puis

${ }^{10}$ RiaOJikken Ongaku Geki: RiaO, de la compagnie Ban'yu Inryoku, collectif qui a succédé à celui de Shuji Terayama (Tenjo Sajiki) après sa mort en 1983. Metteur en scène : J. A. Seazer. Représentation de RiaO pour l'International Shakespeare Association, Tokyo Panasonic Globe, août 1991. Nos plus vifs remerciements vont au professeur Stephen Orgel (Stanford University), qui nous a très aimablement fourni une copie de l'enregistrement vidéo de RiaO réalisé lors de cette représentation, ainsi que le synopsis de l'œuvre en anglais. 
vient l'entêtement de Lear qui, après avoir remis sa part du royaume à Regan, insiste pour obtenir une réponse de Cordelia (« Nothing will come of nothing. Speak again », I.i.9o). Lear est au centre de la scène, sur un trône surélevé ; devant lui sont agenouillés (le dos à la salle) les personnages de la Cour et, au premier plan, Cordelia, également de dos. À gauche, Regan est debout au début du passage, et un peu plus tard, on voit également Goneril qui occupe une place symétrique à celle de Regan à droite ${ }^{11}$.

Cet extrait est représentatif de RiaO dans son recours à des conventions qui appartiennent pour certaines au théâtre nô, pour d'autres au kabuki, et qui pour d'autres sont dérivées plus généralement des conventions du théâtre traditionnel japonais. Du kabuki, on reconnait le grimage des acteurs (à base de poudre de riz), le symbolisme des couleurs : Regan et Goneril sont en noir et violet, le violet étant dans le théâtre kabuki la couleur des traîtres, tandis que Cordelia a une coiffure qui rappelle celle de la jeune première, avec deux fleurs stylisées de part et d'autre. Mais surtout, l'inspiration pour l'expression corporelle et l'utilisation de la musique est celle du nô et du kabuki. La démarche glissée, les postures stylisées de personnages, l'immobilité expressive des acteurs montrent que, comme dans le nô, c'est au langage du corps, plus qu'à la mimique, qu'est confiée l'expression des sentiments et des émotions.

De même, la fonction de la musique est d'accompagner et de souligner le déroulement de l'action et la diction du texte comme dans le théâtre japonais traditionnel, ce que permettent la domination du rythme et une pulsation fluctuante. Il faut noter que, comme dans le cas de la musique, les éléments inspirés du théâtre japonais traditionnel sont souvent des éléments structurels, et que le théâtre de référence est le nô. Ainsi, RiaO est divisé en ketsu (divisions, séquences), qui ne sont pas toujours caractérisées par l'entrée ou la sortie des personnages, mais plutôt par le genre (danse, solo opératique, scène de théâtre) ou encore par le style musical employé. L'œuvre obéit également au principe du jo-ha-kyu, qui prône l'élasticité du rythme au niveau de la structure générale comme à l'intérieur des scènes. Ainsi, le début de l'œuvre est lent. Il s'agit d'un prologue dansé en deux tableaux, intitulé «A Festival of Fools or a Shadow Purification Ceremony ». Il est suivi d'un passage central qui

\footnotetext{
${ }^{11}$ Le passage analysé correspond à la scène 4 de RiaO (16’38” à 18'50").
} 
comprend tout le reste du synopsis et l'essentiel de la partie qui n'y figure pas parce qu'elle a été créée sur scène. Le passage central est en accélération constante jusqu'à un paroxysme, le désespoir de Lear devant le corps de Cordelia, lequel se situe au début de la partie semiimprovisée de l'œuvre. Le final est court et consiste en une série de danses et de pantomimes.

Cependant, les références à ce théâtre traditionnel ne sont pas homogènes. C'est beaucoup d'y trouver des genres très différents comme le nô ou le kabuki. Mais la stratification générique ne s'arrête pas là, puisque RiaO emprunte non seulement au Japon traditionnel, mais encore au Japon moderne. En témoigne notamment l'emploi de la danse buto, une danse expérimentale créée dans les années 1950 et 1960, utilisée dans le prologue de $\mathrm{RiaO}^{12}$.

Ce prologue est bipartite et s'ouvre sur la première danse de l'œuvre $^{13}$. A l'arrière-plan, on distingue un danseur buto dans un halo de lumière bleue ${ }^{14}$. La symbolique de la danse buto est instrumentalisée pour introduire les thèmes directeurs de RiaO (où on reconnaît une lecture attentive de King Lear), l'anéantissement, l'enténèbrement, la violence. Cette symbolique découle de l'esthétique propre à la danse buto, ou " danse des ténèbres ", esthétique du refus, de la contestation, née du refus de la danse européenne et de la volonté de retrouver l'essence du Japon pré-moderne à travers une référence à l'homme primitif, au vide créateur et destructeur, et à une violence chargée d'une fonction cathartique ${ }^{15}$. Vicki Sanders insiste sur le caractère contestataire de cette danse :

Hijikata developed a style of choreographic extremism he called ankoku buto, interpreted to mean "dance of darkness » [...] a term later abbreviated simply to buto. He built his new dance using the reprobates' crude physical features and uncouth habits, a direct assault on the refinement (miyabi) and understatement (shibui) so valued in Japanese

${ }^{12}$ Le passage analysé correspond à la scène $\mathrm{n}^{\circ} 1$, «A Festival of Fools or a Shadow Purification Ceremony » (7'07" à 8'52").

13 La danse est partout dans RiaO, elle est à la fois dans et entre les scènes (cf. synopsis), et elle revêt une importance majeure dans la structuration symbolique de l'œuvre par les paramètres non textuels.

14 Voir Douglas Kerr, op. cit., p. 165.

15 Comme l'explique Susan Blakely Klein (éd.), Ankoku Buto: The Premodern and Postmodern Influences on the Dance of Darkness, 1988, p. 142-147. Voir aussi Vicki Sanders, "Dancing and the Dark Soul of Japan : An Aesthetic Analysis of Buto », Asian Theatre Journal 5 (2) 1988, p. 148-163. 
aesthetics [...]. Hijikata turned away from the Western styles of dance, ballet and modern, that have predominated in twentieth-century Japan. He felt the Japanese body was not made for such movement, that the imported forms did not fit the substance of Japanese life ${ }^{16}$.

Cette esthétique se traduit par les postures torturées du corps, la tension constante des attitudes, la position de base souvent près du sol, le corps nu et peint en blanc (le danseur de RiaO est vêtu d'un simple pagne).

À la fin de cette danse buto, Cordelia, vue comme symbole du désespoir mais aussi du renouveau, apparaît lentement au premier plan ; par son cri, elle fait la transition avec la deuxième partie, où des danseurs évoluent sur une musique d'un style qui contraste violemment avec la mélodie lente et répétitive accompagnant la danse buto. Ces danseurs ont les yeux bandés, et ôtent leurs bandeaux, référence au thème de l'aveuglement mais aussi à la fonction de l'œuvre, qui est de permettre une catharsis, une « purification » comme l'indique le titre du prologue.

Ces deux exemples sont représentatifs de l'esthétique de RiaO, où est érigée en principe structurel la juxtaposition de genres très différents, traditionnels et modernes. En ce sens, les éléments japonais font partie en eux-mêmes d'une esthétique postmoderne dont le premier niveau de lecture (pour le spectateur occidental) est la superposition d'éléments culturels hétérogènes.

C'est dans ce cadre que s'inscrivent non seulement les références au théâtre, à la musique et à la danse japonais, mais aussi les multiples emprunts à la culture théâtrale et musicale européenne. L'influence esthétique du "théâtre de la cruauté » d'Artaud est le premier en importance comme on le verra plus loin. Autre emprunt, la danse : l'utilisation structurelle et symbolique de la danse dans RiaO, qui vient du théâtre traditionnel japonais, recouvre des types de danse aussi différents que la danse buto et la danse européenne moderne. De même, la musique se déploie dans un cadre qui est celui du théâtre japonais traditionnel, mais elle est de style rock.

Cette stratégie du bariolage, présente aussi dans Rock Opera Hamlet, s'avère l'agent d'un contraste permanent et d'une torsion

16 Vicki Sanders, art. cit., p. 149 \& 152. 
mutuelle des éléments endogènes et exogènes qui reflètent, à travers Shakespeare, les tensions du Japon moderne.

RiaO et Rock Opera Hamlet reflètent l'idée que la réception des œuvres de Shakespeare contribue à la perception et à la critique de l'identité japonaise moderne. Comme l'observe le critique japonais Yoshihara Yukari :

The reception of Shakespeare's works contributed, in its small way, to the construction of the modern Japanese nation-state, for it offered opportunities to invent an imaginary Japaneseness through partial and often mutually contradictory identification with and differentiation from the west, as represented by Shakespeare ${ }^{17}$.

L'hétérogénéité de Ria $O$ et de Rock Opera Hamlet est en fait un outil de réflexion sur le Japon moderne et son rapport à la culture occidentale comme à sa propre culture traditionnelle, celle qui a subi la domination de l'Occident d'après-guerre, et surtout à partir des années 1960 (un exemple spectaculaire est l'enseignement de la musique traditionnelle japonaise, qui a été remplacé par celui de la musique occidentale dans les écoles et les universités, à de rares exceptions près $\left.{ }^{18}\right)$. La réponse des modèles esthétiques japonais a été de deux sortes - une esthétique du refus, surtout de l'individualisme et du consumérisme, notamment dans le « nouveau théâtre » japonais dont Terayama, l'inspirateur de RiaO, a été l'instigateur ${ }^{19}$; et simultanément, des tentatives de refondation adossées aux genres artistiques traditionnels - comme dans la danse buto.

${ }^{17}$ Yoshihara Yukari, «Japan as 'half-civilized': an early Japanese adaptation of Shakespeare's The Merchant of Venice and Japan's construction of its national image in the late nineteenth century », in Performing Shakespeare in Japan, op. cit., p. 21-22.

18 Voir Akira Tamba, La musique classique du Japon du XVe siècle à nos jours, Paris, Publications Orientalistes de France, 2001, p. 157. L'auteur précise qu'il existe de nos jours quelque « 83 universités et départements musicaux et 74 lycées musicaux où l'on enseigne la musique occidentale classique», mais seulement de "rares universités qui, comme Takasaki, Seiha, ou Tôkai, enseignent la musique traditionnelle ». La musique traditionnelle japonaise " a survécu grâce à l'enseignement direct de maîtres à disciples qui s'est perpétué parallèlement ».

${ }^{19}$ L'œuvre de Terayama illustre la façon dont ces mutations ont brutalement mis en question les structures socioculturelles traditionnelles et provoqué des mouvements de rejet et des tentatives d'assimilation qui se sont toutes deux traduites, dans le monde de l'art (notamment pour le théâtre et la musique), par des changements esthétiques de grande ampleur fondés sur le constat d'une rupture avec le passé et la recherche d'une nouvelle cohérence. Carol Sorgenfrei, Unspeakable Acts: The Avant-Garde Theatre of Terayama Shuji in Postwar Japan. Honolulu, University of Hawai Press, 2005, chap. 1 : «Cultural Outlaw in a Time of Chaos », p. 17-26. 
Dans Rock Opera Hamlet, la contestation se traduit par le malêtre prêté au personnage éponyme. D'ailleurs, cet opéra rock est encore plus centré sur Hamlet que la tragédie de Shakespeare, et d'une façon qui vise à détailler ses états d'âme. L'hésitation de Hamlet à venger son père devient prétexte à multiplier les chansons qui évoquent son désarroi existentiel et ses peines de cœur. Ainsi, ses monologues de l'acte I.ii et de l'acte II.ii fournissent-ils la matière de la chanson poétiquement intitulée « Life is shit !! », le monologue de III.i est repris au moins pour le titre dans le solo " To Be or Not to be », la relation de Hamlet avec Ophélie devient un amour partagé, larmoyant, mais impossible tant que Hamlet n'a pas accompli sa vengeance (trois chansons sont consacrées à ce sujet), le rôle de Horatio comme confident est considérablement développé (ainsi Hamlet lui confie -t-il son amour pour Ophélie). Le recentrage sur Hamlet est d'autant plus flagrant qu'il se fait au détriment du reste de la pièce, dont des pans entiers disparaissent - de la relation avec Gertrude à la Souricière en passant par le pauvre Yorick.

Cette interprétation de Hamlet est tout à fait représentative de l'inféodation du texte shakespearien à la construction symbolique de l'identité japonaise dans les adaptations récentes de la tragédie. Comme l'observe le critique Kazuko Matsuoka à propos des mises en scène japonaises de Hamlet dans les années 1990 :

Instead of searching out new interpretations of a classic, their approach has been to use Hamlet as a vehicle to project their own particular problems or concerns. Instead of simply representing Shakespeare's text, they have presented adaptations which depict contemporary troubles by alluding to those described in the original play. [...] A Hamlet who speaks contemporary Japanese is a mirror which can reflect the troubles of our own world ${ }^{20}$.

${ }^{20}$ Kazuko Matsuoka, « Metamorphosis of Hamlet in Tokyo ", in Hamlet and Japan, op. cit., p. 230 , p. 233 . 
Dans Rock Opera Hamlet ${ }^{21}$, c'est le courant musical choisi qui permet d'exprimer à travers Hamlet un mal-être face au monde moderne. Le groupe Penicillin appartient en effet au courant du visual $k e i$, né en 1988, qui regroupe des types de musique différents (heavy metal, punk hardcore, darkwave), mais dont le facteur commun, un rejet des valeurs conventionnelles, se traduit par une esthétique de la violence qui vise à provoquer un choc chez le spectateur ${ }^{22}$. D'où une musique et une gestuelle généralement agressives, une apparence androgyne, ou encore des coiffures, des costumes et un maquillage grotesques ou d'inspiration néo-gothique ${ }^{23}$. On peut noter que le groupe à l'origine du visual kei, X-Japan, a également proposé un Rock Opera Hamlet (1996), contemporain de celui de Penicillin, et dans un style similaire. L'influence du courant néo-gothique, sensible dans tout l'opéra à travers l'utilisation de l'orgue électronique et les citations musicales du Dies Irae, est également signifiante, puisque la musique néo-gothique est associée à un message violemment contestataire.

Dans RiaO, l'idée de contestation se traduit par la bigarrure des styles et des influences, par la référence essentielle au genre traditionnel du nô, et par la violence de l'ensemble. Mais surtout, elle s'appuie sur le «théâtre de la cruauté » d'Artaud, source d'inspiration

${ }^{21}$ Hamlet étant depuis l'ère Meiji la pièce de Shakespeare qui reflète le plus massivement l'évolution des formes dramatiques japonaises au contact du théâtre occidental, c'est aussi la pièce de Shakespeare qui reflète de façon privilégiée les mutations du Japon devant la modernisation. Cette interprétation de la tragédie élisabéthaine comme miroir de problèmes socioculturels japonais hérite d'une longue tradition au Japon. Voir Yasunari Takahashi, "Hamlet and the Anxiety of Modern Japan ", Shakespeare Survey 48 (1995), p. 15, où l'auteur expose le point de vue de Tokoku Kitamura, poète de la fin du XIX ${ }^{\mathrm{e}}$ siècle : " He believed in drama as a mirror up to the age; he criticized kabuki-influenced contemporary drama for its lack of great themes such as the fate of man, the meaning of death, or the mystery of the universe [...] he aspired to give theatrical expression to what he called in his celebrated essay "the inner life" of modern self, which could have been an echo of what Hamlet had called "that within which passes show" ". Voir aussi Hiroshi Izubuchio, "A Hamlet of Our Own: Some Japanese Adaptations ", in Hamlet in Japan, op. cit., p. 187-202.

22 Pour une description plus détaillée des genres musicaux populaires japonais contemporains, notamment le visual kei, voir Timothy J. Craig, Japan Pop! Inside the World of Japanese Popular Culture, Armonk, New York, M. E. Sharpe, 200o. Comme phénomène socioculturel, le visual kei est également mentionné par Bonnie C. Wade dans "Music in Japan : Experiencing Music, Expressing Culture », Journal of the Society for Ethnomusicology, 50 (33), 2006 Fall, p. 505.

23 Yasser Mattar, « Miso soup for the ears : Contemporary Japanese Music and its Relation to the Genres Familiar to the Anglophonic Audience », Popular Music and Society (31:1), Feb. 2008, 113-123, p.117. Voir aussi Japanese Popular Music, op. cit., p.56-58, et les sites http://www.visualkei.biz/ et http://www.escale-japon.com/articles/visual/visual.php. 
essentielle pour Terayama et ses disciples (dont le metteur en scène de RiaO, A. J. Seazer). Comme chez Artaud, le théâtre dans RiaO n'a pas une fin théâtrale, il doit démystifier et libérer la réalité cachée ou refoulée par les préjugés de la société. La violence n’y est que l'expression d'une "catharsis à l'envers », un « exorcisme pour faire affluer nos démons », comme le formule Henri Gouhier à propos du théâtre d'Artaud :

« Tout cela semble un exorcisme pour faire affluer nos démons. » Image remarquable : exorciser, c'est, par définition, chasser les démons; à l'Exposition coloniale, Artaud voit clairement, à la lumière d'un éblouissant paradoxe, ce qu'il a toujours pensé du théâtre : il est un exorcisme à rebours ${ }^{24}$

La violence sur scène n'est qu'un aspect, le plus apparent, de la métaphysique d'Artaud, dans laquelle :

le théâtre ou plutôt son théâtre est le langage propre à l'expression des puissances obscures que sa métaphysique voit dans la nature; il est donc, par vocation, le lieu où le mal inhérent à leur être se manifeste, terrifiant ${ }^{25}$..

Dans ce cadre, le partage du royaume de Lear n'est qu'un des éléments signifiants, de même que King Lear dans son ensemble ne fournit que la source des thèmes de l'aveuglement, de la violence, de l'anéantissement, dont la portée idéologique est indiquée par l'amoncellement de paramètres non textuels et symboliques.

L'inféodation de l'œuvre shakespearienne à des problématiques purement nipponnes dans Rock Opera Hamlet est donc également présente dans RiaO. Mais elle est prise dans la conception artésienne du «théâtre total », et par là dans un paradoxe qui n'est pas le moins fascinant de RiaO. Nous avons vu que l'esthétique du bariolage produite par la rencontre des éléments japonais et européens faisait partie de l' " exorcisme » recherché dans RiaO. Or, non seulement c'est une œuvre européenne qui fournit une partie des cadres esthétiques de l'œuvre, mais de plus il existe une réelle continuité entre certains éléments japonais et les principes directeurs d'un théâtre contestataire certes, mais européen. Ainsi de la continuité symbolique entre la danse buto et le «théâtre de la cruauté », qui a été démontrée par Vicki

24 Henri Gouhier, Antonin Artaud et l'essence du théâtre, Paris, Vrin, 1974, p. 92.

25 Ibid., p. 93. 
Sanders dans son article sur « l'âme sombre du Japon ». Elle explique que la danse buto vient du refus de l'influence occidentale, en l'espèce, de la danse classique européenne : mais que, dans sa recherche d'une libération des forces de l'inconscient et d'une énergie primitive, la danse buto (ou «danse des ténèbres ») prolonge la «catharsis à l'envers » du théâtre d'Artaud ${ }^{26}$, en particulier pour extérioriser la violence sociale. C'est dans ce sens qu'elle est utilisée dans RiaO : les « forces obscures » de l'homme doivent se libérer à travers une vitalité puissante, impersonnelle et effervescente, faisant partie d'un théâtre qui comme celui d'Artaud « est fait pour vider collectivement des abcès » et qui doit permette l'éclosion de «forces irrationnelles » qui « donnent droit de cité et d'existence à des actes hostiles par nature à la vis des sociétés ${ }^{27}$ ». D'autre part, il existe une connivence entre certaines caractéristiques du théâtre japonais, où le texte n'est traditionnellement qu'un des paramètres, en raison de l'importance de la musique et de la danse, et le « théâtre total » voulu par Artaud et luimême influencé par le théâtre japonais traditionnel, comme l'observe Florence de Mèredieu :

En 1921, Artaud écrit à Max Jacob: "Les Japonais sont nos maîtres directs, et nos inspirateurs. » Son idéal est alors l'acteur japonais qui joue sans accessoires, et dont le jeu est suprêmement intériorisé. [...] Les Japonais exercent [...] une influence considérable sur les conceptions scéniques de l'auteur du "Théâtre Alfred Jarry », comme sur la rédaction de cet ouvrage fondateur que demeure Le Théâtre et son double ${ }^{28}$.

Le jeu des influences mutuelles entre Artaud et RiaO constitue donc pour ce dernier un retour paradoxal aux sources du théâtre japonais, qui, vu à travers le prisme artésien, contribue à la réinvention du langage théâtral voulue par Terayama et ses successeurs. En effet, comme le rappelle Mèredieu, le dramaturge avait particulièrement retenu du théâtre japonais traditionnel la relation de la scène et de la salle ainsi que le jeu de l'acteur, qui contribuent tous à la définition

\footnotetext{
${ }^{26}$ Vicki Sanders, art. cit., p. 149-150. L'auteur cite Le théâtre et son double comme introduction à l'esthétique de la danse buto, et observe : "Artaud's description cuts to the heart of Japan's dance of darkness, with its eerie, mutantlike qualities, its crudeness and freakish anthropomorphism. »

${ }^{27}$ Henri Gouhier, op. cit., p. 97.

28 Florence de Mèredieu, Le Japon d'Antonin Artaud / La Chine d'Antonin Artaud, Paris, Blusson, 2006, p. 24.
} 
artésienne du spectacle intégral. Dans sa «Quatrième Lettre sur le langage », Artaud écrit d'ailleurs :

C'est ainsi que dans le théâtre oriental, il n'y a pas de langage de la parole, mais un langage de gestes, d'attitudes, de signes, qui au point de vue de la pensée en action a autant de valeur expressive et révélatrice que l'autre ${ }^{29}$.

Dans le théâtre d'Artaud, selon Henri Gouhier, «l'œuvre théâtrale est d'abord une action dramatique, tous les moyens d'expression étant à son service, chacun exprimant ce qui ne saurait l'être par les autres. Ceci exclut, bien sûr, la suffisance du texte ${ }^{30}$ », au sens où le mot ne doit pas redire tout ce que peuvent exprimer la lumière ${ }^{31}$, le geste, la couleur, le mouvement, le bruit ou le silence ».

De même, dans RiaO, la danse n'est pas seulement ajoutée à la fin de certaines scènes ou entre les scènes : elle se substitue au texte lui-même à plusieurs reprises. Le début de l'acte II de King Lear fait ainsi disparaitre Curan, qui a pour rôle d'informer Edmond de la venue de Cornouailles et de Regan. Il est remplacé par des « danseurs des ténèbres » qui ont une fonction symbolique et emphatique : mettre en relief la traîtrise d'Edmond. Autre passage où la danse se substitue au texte, la séquence « Roses of Sterile Women », où une danse remplace le dialogue qui a lieu entre Goneril et Oswald à l'acte I scène iii, scène dans laquelle Goneril recommande à Oswald d'être aussi désagréable que possible avec Lear, en vue de le chasser de chez elle. Dans RiaO, la scène a une charge symbolique différente. Comme l'indique le synopsis, la danse « révèle la sensualité » de Goneril : elle suggère une sensualité brutale, effrayante, prenant plutôt des allures de combat chorégraphié32 $^{2}$. La bestialité de Goneril est ainsi liée dans RiaO au thème du désir mauvais et irrépressible, qui participe à la « catharsis à l'envers » héritée d'Artaud.

\footnotetext{
29 Ibid., p. 24.

${ }^{30}$ Henri Gouhier, op. cit., p. 139

${ }^{31}$ Les seuls jeux de lumière font littéralement de RiaO une œuvre sombre : la majorité des scènes se déroule dans la pénombre sinon dans le noir complet (comme pour une partie du prologue). Même les couleurs qui dominent l'éclairage quand il y a effectivement de la lumière sur scène, le rouge, l'or et le bleu, ne sont pas des teintes vives, et elles sont comme assiégées par la noirceur en raison des variations de lumière au cours des scènes.

32 Cette analyse correspond à la séquence « Roses of Sterile Women » (33’36” à 35'04”).
} 
Rock Opera Hamlet et RiaO témoignent qu'au Japon aussi, le théâtre de Shakespeare n'est autre que le miroir du monde. L'Extrême-Orient et l'Occident s'y fertilisent mutuellement, dans la rencontre de l'ancien (Shakespeare) et du moderne (la danse buto), du savant (le nô) et du populaire (le rock). Cette bilocation est ainsi l'outil paradoxal d'une continuité entre le Japon pré-moderne et celui d'aujourd'hui, dans des adaptations de Shakespeare qui traduisent la recherche de nouveaux paradigmes culturels au miroir de l'étranger.

Claire BARDELMANN

Université Paul Verlaine (Metz) 